\title{
Efficacy and Safety of Carbon Dioxide Ablative Fractional Resurfacing (CO2-AFR) Device in Moderate to Severe Atrophic Acne Scars
}

\author{
Wajieha Saeed, ${ }^{1}$ Zahida Rani, ${ }^{2}$ Shazia Aslam $^{3}$
}

\begin{abstract}
Acne having a prevalence of $>90 \%$ in adolescents persisting in adulthood, causes inflammation and scaring. Acne scars cause severe social, psychological and cosmetic problems. There are many remedies available for acne scars with variable results. CO2-AFR laser is a new modality with more effectiveness and low side effect profile.

Objective: To determine the effectiveness and safety of CO2-AFR device in the treatment of moderate to severe atrophic acne scars.

Study Design: Interventional study.

Place and Duration of Study: This study was conducted at Dermatology Department Unit-I, Mayo Hospital Lahore and duration was 1 year, from April 2015 to March 2016.

Material and Methods: The 30 patients of either sex, having moderate to severe atrophic acne scars were enrolled in the study. Pre-treatment assessment of acne

\footnotetext{
${ }^{1}$ Senior Registrar, Dermatology Department, Mayo Hospital, Lahore

${ }^{2}$ Assistant Professor, Dermatology Department, Mayo Hospital, Lahore
}

${ }^{3}$ Consultant Dermatologist, Najran University, Saudi Arabia
\end{abstract}

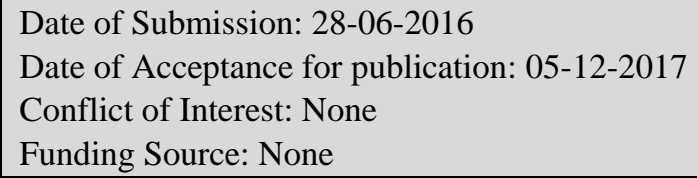

\section{Contribution}

All Authors have contributed in Study Design, Data Collection, Data Analysis, Data Interpretation, Manuscript Writing and Approval. scars was done by using Goodman \& Baron qualitative scarring grading system. Patients were treated at $4-5$ weeks interval with maximum 5 sessions. Objective assessment for efficacy and side effects was done at each session. Data was collected on a specially designed proforma and statistical analysis carried out by using SPSS version 17.

Results: Significant improvement in acne scars was observed. 14 (46.7\%) patients showed good efficacy (50-75\% reduction), 1 (3.3\%) patient came out with excellent efficacy $(>75 \%)$ and $14(46.7 \%)$ patients showed fair efficacy $(25-50 \%$ reduction in acne scars). $13.3 \%$ patients showed few delayed side effects which resolved with treatment.

Conclusion: CO2-AFR device was proved to be effective and safe in the treatment of moderate to severe atrophic acne scars.

Keywords: Atrophic acne scars, ablative fractional resurfacing (AFR), CO2 laser, fractional photothermolysis (FP).

\section{Introduction}

Acne has a prevalence of $>90 \%$ in adolescents, ${ }^{1}$ persisting in adolescence in almost $12 \%-14 \%$ of cases with severe social, psychological and cosmetic implycations. $^{2}$ All areas of body having high concentration of pilosebaceous glands are involved, but acne is more prevalent over face, back and chest. Acne lesions can be inflammatory and non-inflammatory. Lesions with inflammation can lead to scarring. ${ }^{3}$

The pathogenesis of acne is multifactorial, such as excessive sebum production, raised androgen levels or increased sensitivity of their end receptors, propionibacterium acnes proliferation and hyper-keratinization 
of follicular epithelia. ${ }^{4}$

Free fatty acids are produced, as a result of enzymes released from bacteria, which lead to inflammatory lesions. These inflammatory lesions can heal with scarring. Acne scars can be atrophic $(89-90 \%)$ and hypertrophic. ${ }^{5}$ There are several classifications of acne scars, whereby scars are named as rolling, icepick, shallow boxcar and deep boxcar. ${ }^{5}$ Rolling scars are Mshaped, gently undulating like hills and valleys withoutsharp borders. Icepick scars are V-shaped, also known as pitted scars, appear as round, deep depressions with a pinpoint base. Boxcar scars are U-shaped, usually round, polygonal or linear at the skin surface. Boxcar scars are the most common type, followed by rolling and icepick scars.,

Various procedures like chemical peeling, dermabrasion, punch grafting, fillers and laser resurfacing have been tried to treat acne scars, with variable outcomes. ${ }^{5}$ Among lasers, $\mathrm{CO} 2$ ablative laser has proved to be an effective device for treating atrophic acne scars, ${ }^{6}$ but it is associated with increased risk of undesired effects like edema, erythema, burning, milia, folliculitis, flare-up of acne and pigmentary alterations. ${ }^{6,7}$

To reduce the disadvantages of ablative resurfaceing, fractional photothermolysis (FP) has been introduced. A novel ablative 30WCO2 laser device uses a technique called AFR, combingCO2 ablation with a FP system. FP induces zones of microthermal wounds with surrounding normal tissue, causing rapid re-epithelization. Different studies have shown that by combining ablative technology with FP, AFR treatment produces a well-tolerated and efficacious treatment option for acne scars. ${ }^{5,6}$

\section{Methods}

An analytical experimental study was carriedout in Dermatology Department in a public sector hospital, Lahore and duration was one year from April, 2015 to March, 2016. Thirty patients were recruited for the study by non-probability purposive sampling technique.

Patients of either sex, ages between 20 - 40 years, with skin prototype IV and V, having moderate to severe atrophic acne scars were enrolled in the study. Patient taking any treatment for acne scars for last 3 months, those having active or history of herpes simplex infection, having keloidal tendency or pregnancy were excluded from the study.
After approval by research and ethical committee and written informed consent by patients, complete history was taken and examination carriedout and all the demographic data were recorded on a pre-designed proforma.Pre-treatment assessment of atrophic acne scars severity was done using Goodman and Baron qualitative scarring grading system. ${ }^{8}$ Patients were treated at $4-5$ weeks interval with a maximum of $5-$ sessions. Objective assessment was obtained at each session and graded according to percentage reduction in acne scars on a 4 - point scale as excellent $(>75 \%$ reduction), good $(50-75 \%$ reduction $)$, fair $(25-50 \%$ reduction) and poor $(<25 \%$ reduction). At each session any adverse effects were noted.Digital photographs were taken at baseline, at each session and at 4 weeks follow up after the last session and were compared.All this information was recorded on specially designed proformas. Statistical analysis of the data was done by using SPSS version 17.0.

\section{Results}

The mean age of the patients was $23.8 \pm 2.2$ years. There were $16(53.3 \%)$ female and $14(46.7 \%)$ male patients. $6(20 \%)$ patients were graded as having moderate atrophic acne scars and rest $(80 \%)$ were with severe acne scars. $17(56.7 \%)$ patients had Fitzpatrick's skin type IV and $13(43.3 \%)$ patients were with skin type $\mathrm{V}$.

$23(76.7 \%)$ patients were in the age range of $18-$ 25 years while $6(20 \%)$ were of $26-35$ years of age and $1(3.3 \%)$ patient was 37 years old. In this study, 14 $(46.7 \%)$ patients showed good efficacy $(50-75 \%$ reduction), same number of patients showed fair efficacy $(25-50 \%$ reduction) and $1(3.3 \%)$ patient came out with excellent efficacy i.e. $>75 \%$ reduction in acne scars.

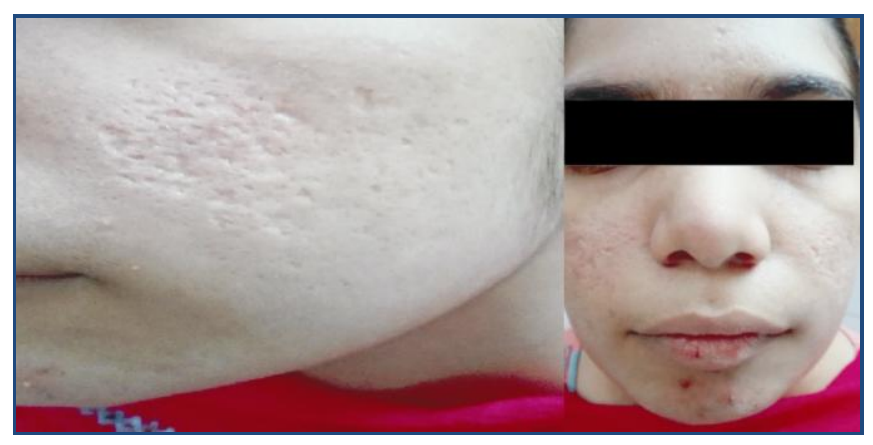

Fig. 1a: Before (Severe Acne Scars). 


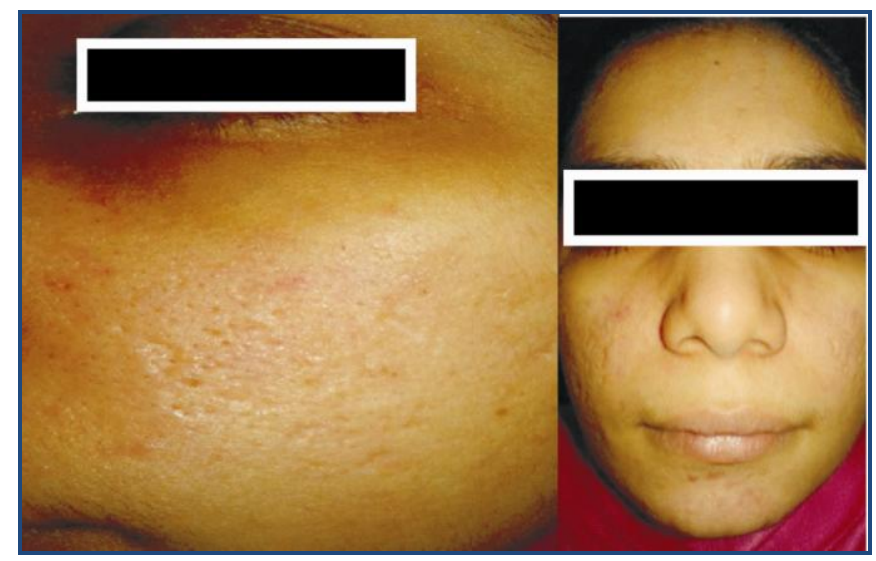

Fig. 1b: After Laser Sessions (Good Efficacy).

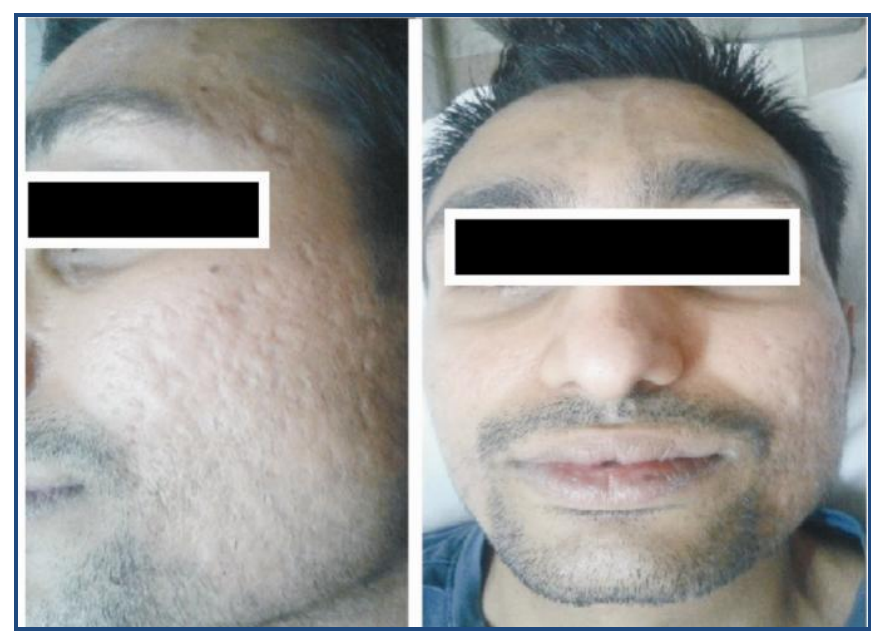

Fig. 2a: Before (Severe Acne Scars).

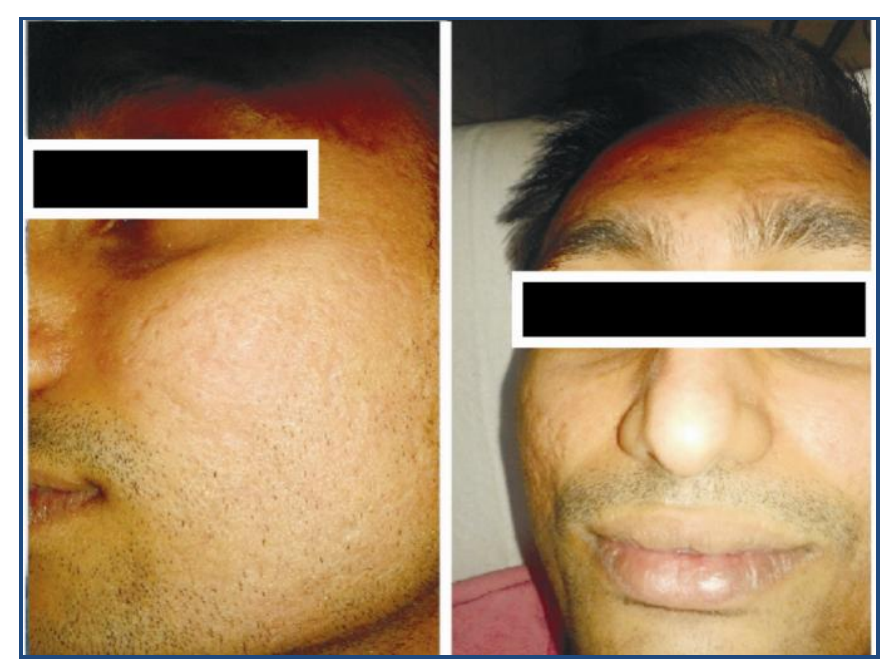

Fig. 2b: After Laser Sessions (Good Efficacy).
The laser parameters like energy, density and depth of ablation was adjusted at each session, according to the severity of acne scars and patient's skin type. Fluence (energy pulse) varied from $15 \mathrm{~mJ} / \mathrm{cm} 2$ to $24 \mathrm{~mJ} / \mathrm{cm} 2$, density level of 2 to 4 and depth level of 37 were used.

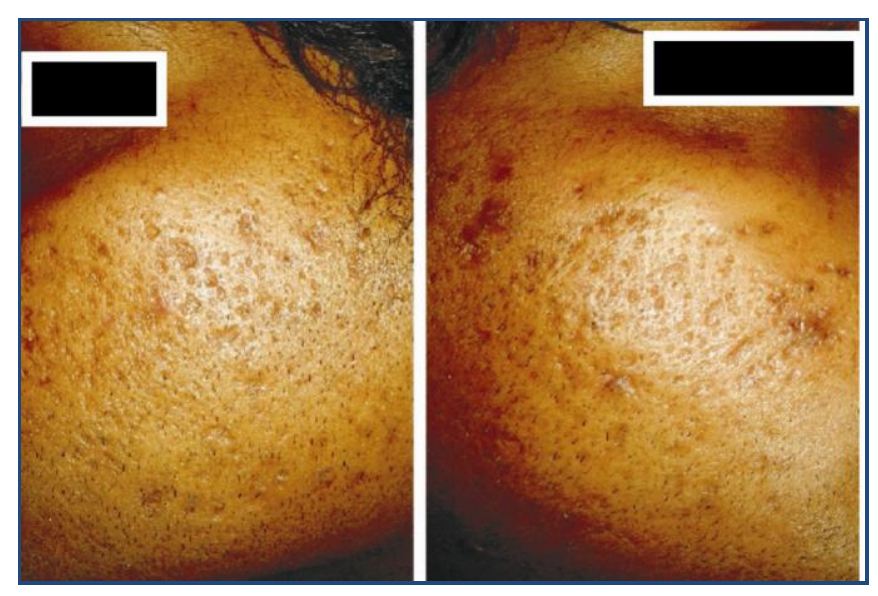

Fig. 3a: Before (Severe Acne Scars).

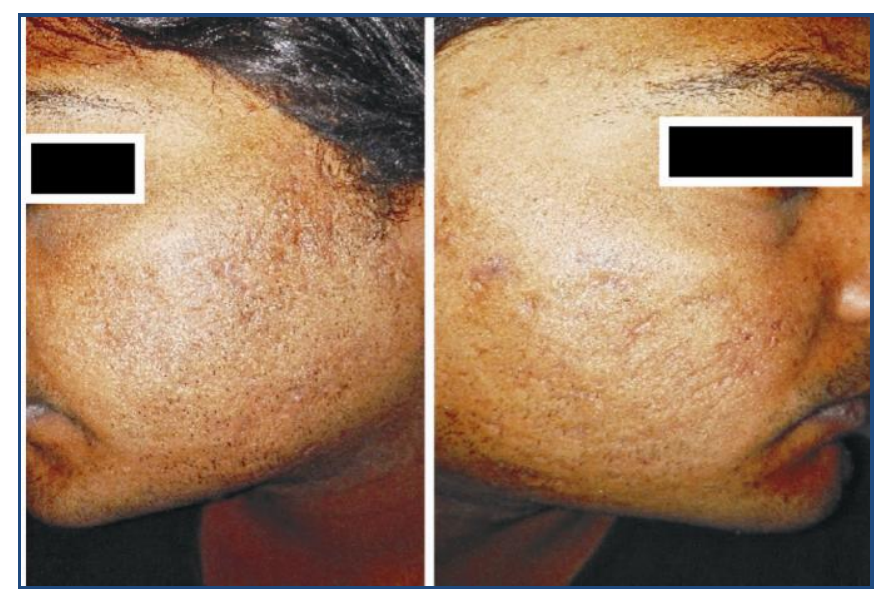

Fig. 3b: After Laser Sessions (Excellent Efficacy).

The stratification of efficacy with grade of scars (Table 1), type of skin (Table 2), sex of patient (Table 3 ) and age of patient (Table 4) is shown below.

Side effects including temporary erythema, edema, burning, and mild superficial crusting were seen in almost all patients, which cleared within few days with topical antibiotic and sunblock application. 7 (23.3\%) patients presented with flare up of acne, which improved with topical and oral antibiotics. 4 (13.3\%) patients showed post-inflammatory hyperpigmentation and milia formation. Both of them had type $\mathrm{V}$ skin 
with severe acne scars. Treatment was discontinued till the clearance of pigmentation with sunblock and topical depigmenting agents, in around 4-5 months. Laser sessions were completed with low fluence and there was no recurrence of pigmentation.

\section{Discussion}

Acne scaring have complex pathogenesis, involving infra-infundibular inflammatory process, follicular rupture and perifollicular abscess formation, all of which stimulates a series of wound healing events. Impaired healing of damaged tissue in and around pilosebaceous unit leads to atrophic or hypertrophic acne scars. ${ }^{5,6}$

Laser skin resurfacing has revolutionized the treatment of atrophic acne scars. Ablative lasers results in destruction of the scar tissue through melting and evaporization. $\mathrm{CO} 2$ laser and Erbium YAG laser are the frequently used ablative lasers for the treatment of acne scars. Non ablative lasers do not cause destruction of the tissue, but stimulate formation of new collagen and results intightening of skin, thus scar being raised to the surface. Among the nonablative lasers, Nd:YAG and Diode are the most commonly used lasers. ${ }^{9,10}$ AFR combines the increased efficacy of ablative techniques with the safety and reduced downtime of FP. ${ }^{11,12}$

The results of our study is comparable with other national and international studies. In study by Simin et al, ${ }^{13} 85 \%$ patient showed $30-70 \%$ reduction in acne scars which is comparable with our study in which $96.7 \%$ patients showed $25-80 \%$ reduction in acne scars. In study by Chan et al, ${ }^{14} 86 \%$ patient showed good and fair efficacy. In study by Manuskiatti et al, ${ }^{15} 85 \%$ patients showed more than $25 \%$ reduction in acne scars. In another study by Imran et al $^{16} 26$ patients $(43.3 \%)$ showed excellent response, 15 patients $(25 \%)$ showed good efficacy and $19(31.7 \%)$ patients showed poor response to $\mathrm{CO} 2$ ablative laser. This difference in results could be due to higher sample size (60 patients), with Fitzpatrick's skin type III and IV and total of $3-4$ sessions on each patient. Our study was also comparable with the national study by Tahir et al, ${ }^{17}$ in which $71 \%$ overall improvement in acne scars was observed.

Adverse effects seen were not significant and only $13.3 \%$ (4) patients showed long-term side effe- cts in the form of post-inflammatory hyperpigmentation and milia formation and $23.3 \%$ (7) patients presented with flare up of acne, which improved with oral and topical therapy in next few weeks to months.

Table 1: N* Number of patients.

\begin{tabular}{|l|l|r|c|}
\hline Grade of Scars & \multicolumn{1}{|c|}{ Efficacy } & \multicolumn{1}{|c|}{$\mathbf{N}^{*}$} & \% of Total N \\
\hline \multirow{3}{*}{ Moderate } & Excellent $>75 \%$ & 1 & $3.3 \%$ \\
& Good 51-75\% & 5 & $16.7 \%$ \\
& Total & 6 & $20.0 \%$ \\
\hline \multirow{5}{*}{ Severe } & Fair 25-50\% & 14 & $46.7 \%$ \\
& Good 51-75\% & 9 & $30.0 \%$ \\
& Poor $<25 \%$ & 1 & $3.3 \%$ \\
& Total & 24 & $80.0 \%$ \\
\hline \multirow{5}{*}{ Total } & Excellent $>75 \%$ & 1 & $3.3 \%$ \\
& Fair 25-50\% & 14 & $46.7 \%$ \\
& Good 51-75\% & 14 & $46.7 \%$ \\
& Poor $<25 \%$ & 1 & $3.3 \%$ \\
& Total & 30 & $100.0 \%$ \\
\hline
\end{tabular}

Table 2: $\mathrm{N}^{*}$ Number of patients.

\begin{tabular}{|l|l|c|c|}
\hline Type of skin & \multicolumn{1}{|c|}{ Efficacy } & \multicolumn{1}{|c|}{$\mathbf{N}^{*}$} & \% of Total N \\
\hline Skin type IV & Excellent $>75 \%$ & 1 & $3.3 \%$ \\
& Fair 25-50\% & 5 & $16.7 \%$ \\
& Good 51-75\% & 11 & $36.7 \%$ \\
& Total & 17 & $56.7 \%$ \\
\hline Skin type V & Fair 25-50\% & \multicolumn{1}{|c|}{9} & $30.0 \%$ \\
& Good 51-75\% & 3 & $10.0 \%$ \\
& Poor $<25 \%$ & 1 & $3.3 \%$ \\
& Total & 13 & $43.3 \%$ \\
\hline Total & Excellent >75\% & 1 & $3.3 \%$ \\
& Fair 25-50\% & 14 & $46.7 \%$ \\
& Good 51-75\% & 14 & $46.7 \%$ \\
& Poor $<25 \%$ & 1 & $3.3 \%$ \\
& Total & 30 & $100.0 \%$ \\
\hline
\end{tabular}


Table 3: $\mathbf{N}^{*}$ Number of patients.

\begin{tabular}{|l|l|r|c|}
\hline \multicolumn{1}{|c|}{ Sex } & \multicolumn{1}{|c|}{ Efficacy } & N* & \% of Total N \\
\hline Female & Excellent $>75 \%$ & 1 & $3.3 \%$ \\
& Fair $25-50 \%$ & 3 & $10.0 \%$ \\
& Good 51-75\% & 11 & $36.7 \%$ \\
& Poor $<25 \%$ & 1 & $3.3 \%$ \\
& Total & 16 & $53.3 \%$ \\
\hline Male & Fair 25-50\% & 11 & $36.7 \%$ \\
& Good 51-75\% & 3 & $10.0 \%$ \\
& Total & 14 & $46.7 \%$ \\
\hline Total & Excellent $>75 \%$ & 1 & $3.3 \%$ \\
& Fair $25-50 \%$ & 14 & $46.7 \%$ \\
& Good 51-75\% & 14 & $46.7 \%$ \\
& Poor $<25 \%$ & 1 & $3.3 \%$ \\
& Total & 30 & $100.0 \%$ \\
\hline
\end{tabular}

Table 4: $\mathbf{N}^{*}$ Number of patients.

\begin{tabular}{|l|l|r|c|}
\hline Age of pt & \multicolumn{1}{|c|}{ Efficacy } & N* & \% of Total N \\
\hline Age 18-25 & Excellent $>75 \%$ & 1 & $3.3 \%$ \\
& Fair 25-50\% & 12 & $40.0 \%$ \\
& Good 51-75\% & 10 & $33.3 \%$ \\
& Total & 23 & $76.7 \%$ \\
\hline Age 26-35 & Fair 25-50\% & 1 & $3.3 \%$ \\
& Good 51-75\% & 4 & $13.3 \%$ \\
& Poor <25\% & 1 & $3.3 \%$ \\
& Total & 6 & $20.0 \%$ \\
\hline Age 36-40 & Fair 25-50\% & 1 & $3.3 \%$ \\
& Total & 1 & $3.3 \%$ \\
\hline \multirow{2}{*}{ Total } & Excellent $>75 \%$ & 1 & $3.3 \%$ \\
& Fair 25-50\% & 14 & $46.7 \%$ \\
& Good 51-75\% & 14 & $46.7 \%$ \\
& Poor <25\% & 1 & $3.3 \%$ \\
& Total & 30 & $100.0 \%$ \\
\hline
\end{tabular}

\section{Conclusion}

$\mathrm{CO} 2$ ablative fractional resurfacing is an effective, well tolerated and safe treatment modality for mode-rate to severe atrophic acne scars in Fitzpatrick's skin type IV and V. Results are better in type IV skin, $56.7 \%$ patients showing good response as compared to type $\mathrm{V}$ skin where $40 \%$ patients showed improvement.

\section{References}

1. Ghodsi SZ, Orawa H, Zouboulis CC. Prevalence, severity and severity risk factors of acne in high school pupils: a community-based study. Journal of Investigative Dermatology. 2009; 129: 2136-41.

2. Capitanio B, Sinegra JL, Bordignon V, Fei PC, Picardo $\mathrm{M}$, Zouboulis CC. Under estimated clinical features of post-adolescent acne. J Am Acad Dermatol. 2010; 63: 702-8.

3. Layton M, Henderson CA, Cunliffe WJ. A clinical evaluation of acne scarring and its incidence. Clin Exper Dermatol. 1994; 19: 303-8.

4. Kurokawa, Danby FW, Jui Q. New developments in our understanding of acne pathogenesis and treatment. Experimental Dermatology 2009; 18: 821-32.

5. Fabbrocini G, Annunziata MC, Arco VD. Acne scars: pathogenesis, classification and treatment," Dermatology Research and Practice", vol. 2010, Article ID 893080, 13 Pages, 2010. http://dx.doi.org/10.1155/2010/893080.

6. FifeD, Clin J. Practical evaluation and management of atrophic acne scars: tips for the general dermatologist. Aesthet Dermatol. 201; 4 (8): 50-7.

7. Tierney EP, Hanke CW. Treatment of $\mathrm{CO} 2$ laser induced hypopigmentation with ablative fractional laser resurfacing: case report and review of literature. J Drugs Dermatol. 2010; 9: 1420-6.

8. Goodman GJ, Baron JA. Post-acne scarring - a quantitative global scarring grading system. J Cosmet Dermatol. 2006; 5: 48-52.

9. Alster TS, Tanzi EL, Lazarus M. The use of fractional laserphotothermolysis for the treatment of atrophic scars. DermatolSurg. 2007; 33: 295-9.

10. Nelson S. "An introduction to laser and laser-tissue interactions in dermatology" in principles and practices in cutaneous laser surgery. A.N.B. Kauvar, Ed., p.70, Taylor and Francis Group, Boca Raton, USA, 2005.

11. Haseganla T, Matsukura T, Mizuno Y. Clinical trial of a laser device called fractional photothermolysis system for acne scars. J Dermatol Treat. 2006; 33: 623-7.

12. Lee Hs, Lee JH, Ahn GY. Fractional photothermolysis for the treatment of acne scars: a report of 27 Korean patients. J Dermatol Treat. 2008; 19: 45-9.

13. Simin S, Azadeh M. Evaluation of fractional $\mathrm{CO} 2$ laser efficacy in acne scar. Lasers Med Sciences. 2013; 3 (2): 56-60.

14. Chan NP, HoSG, Yeung CK, Shek SY, Chan HH. Fractional ablative carbon dioxide laser resurfacing for skin rejuvenation and acne scars in Asians. Lasers Surg Med. 2010; 42 (9): 615-23.

15. Manuskiatti W, Triwongwaranat D, Varothai S. Efficacy and safety of carbon dioxide ablative fractional resurfacing device for treatment of atrophic acne scars in Asians. J Am Acad Dermtol. 2010; 63: 274-83. 
16. Imran M, Saha I. Fractional CO2 laser resurfacing as monotherapy in the treatment of atrophic facial acne scars. Journal of cutaneous and aesthetic surgery. 2014; 7 (2): 87-92.
17. Tahir J, Farhana M, Haroon N, Sarmad M, Ayesha N, Rabiya Y. Efficacy and safety of ablative fractional carbondioxide laser for acne scars. Journal of Pakistan Association of Dermatology. 2012; 22: 41-44. 